Revista Bioética

\title{
PESQUISA
}

\section{Análise de prontuários de hospital universitário de Mogi das Cruzes, São Paulo}

Mariana Cuccato Ribeiro ${ }^{1}$, Bruno Skawinski Dalaneze ${ }^{1}$, Mariah Padovan de Oliveira Peruchi ${ }^{1}$, Raquel Barbosa Cintra ${ }^{1}$

1. Universidade de Mogi das Cruzes, Mogi das Cruzes/SP, Brasil.

\section{Resumo}

O prontuário médico é, na área da saúde, documento básico que permeia toda a atividade assistencial, administrativa, de pesquisa e de ensino, permitindo a comunicação entre os diversos profissionais que participam do cuidado do paciente. Destaca-se, no entanto, a diferença de qualidade dos registros produzidos por cada instituição. Considerando essa variedade, o objetivo desta pesquisa foi analisar prontuários de um hospital de ensino de Mogi das Cruzes/SP. Foram avaliados 191 prontuários, datados a partir de 2016. A avaliação consistiu na aplicação de instrumento que atribui escores considerando os itens obrigatórios do prontuário. Apesar de os escores totais obtidos não serem baixos, os resultados demonstram que a qualidade dos prontuários varia e que o hospital-escola deve aperfeiçoar seu preenchimento.

Palavras-chave: Registros médicos. Educação médica. Códigos de ética.

\section{Resumen}

\section{Análisis de historias clínicas de un hospital universitario de Mogi das Cruzes, São Paulo, Brasil}

La historia clínica es un documento básico en el área de la salud que impregna todas las actividades de asistencia, administrativas, de investigación y de enseñanza, además de permitir la comunicación entre los diversos profesionales responsables de los pacientes. Sin embargo, se destaca la diferencia en la calidad de los registros producidos por cada institución. Considerando esta variedad, el objetivo de esta investigación fue analizar los registros médicos de un hospital docente en Mogi das Cruzes, São Paulo, Brasil. Se evaluaron 191 historias clínicas, fechadas en 2016. La evaluación consistió en la aplicación de un instrumento que asigna puntajes considerando los ítems obligatorios de la historia clínica. Aunque las puntuaciones totales obtenidas no son bajas, los resultados demuestran que la calidad de las historias clínicas varía y que el hospital docente debe mejorar su cumplimentación. Palabras clave: Registros médicos. Educación médica. Códigos de ética.

\section{Abstract}

\section{Analysis of medical records of a university hospital in Mogi das Cruzes, São Paulo, Brazil}

The medical record is a basic document in healthcare that permeates all assistance, administrative, research and teaching activities, in addition to allowing communication between health professionals. However, we should emphasize the difference in the quality of these records produced by different institutions. This study aimed to analyze the quality of this data in a teaching hospital in the city of Mogi das Cruzes, São Paulo, Brazil. A total of 191 medical records dated from 2016 were included in the evaluation, which consisted in applying an instrument that ascribe a score to each obligatory item in the medical record. Although the total scores obtained were not low, the results show that the quality of medical records varies, and that the teaching hospital should improve how they are filled out.

Keywords: Medical records. Education, medical. Codes of ethics. 
O Ministério da Saúde define "hospital" como parte integrante de uma organização médica $e$ social, cuja função básica consiste em proporcionar à população assistência médica integral, curativa e preventiva, sob quaisquer regimes de atendimento, inclusive o domiciliar, constituindo-se também em centro de educação, capacitação de recursos humanos e de pesquisas em saúde, bem como de encaminhamento de pacientes, cabendo-Ihe supervisionar e orientar os estabelecimentos de saúde a ele vinculados tecnicamente ${ }^{1}$. "Hospital geral" é definido como aquele destinado a atender pacientes portadores de doenças das várias especialidades médicas ${ }^{1}$. Já o "hospital de ensino", ou "universitário", seria um hospital geral com as características e funções do hospital de base, utilizado por escolas de ciências da saúde, como centro de formação profissional ${ }^{2}$.

O hospital é organização complexa, e sua estrutura é altamente burocratizada e interdependente. Uma de suas ferramentas é o prontuário médico, que deve propiciar comunicação eficiente entre os diversos profissionais envolvidos no atendimento em saúde. Esse documento tem como função registrar dados fornecidos pelo paciente, resultados de exames médicos e condutas adotadas pelos profissionais. O Conselho Federal de Medicina (CFM), no artigo 1으 da Resolução CFM 1.638/2002, define prontuário médico como o documento único constituído de um conjunto de informações, sinais e imagens registradas, geradas a partir de fatos, acontecimentos e situações sobre a saúde do paciente e a assistência a ele prestada, de caráter legal, sigiloso e científico, que possibilita a comunicação entre membros da equipe multiprofissional e a continuidade da assistência prestada ao indivíduo ${ }^{3}$.

Tavakoli e Jahanbakhsh reiteram que o prontuário médico serve para diferentes propósitos nas operações diárias de uma organização de saúde. Permite que os profissionais se comuniquem uns com os outros; fornece uma base para planejar o tratamento do paciente; documenta a qualidade dos cuidados para revisão futura; fornece informações para análises estatísticas; e estabelece uma base para processos de faturamento e geração de relatórios financeiros ${ }^{4}$.

O Código de Ética Médica (CEM) ${ }^{5}$ norteia a conduta profissional, inclusive relacionada a documentos médicos. Em seu artigo 88, o CEM proíbe o médico de negar, ao paciente, acesso a seu prontuário, deixar de Ihe fornecer cópia quando solicitada, bem como deixar de lhe dar explicações necessárias à sua compreensão $o^{5}$. Trata-se, portanto, de registro pertencente ao paciente, que deve ser elaborado de maneira legível, como ressalta o artigo 87 do mesmo código ${ }^{5}$.
Como é usado também para fins jurídicos, o prontuário deve estar sempre atualizado. É por meio dele que se pode saber se os cuidados aplicados foram corretos ou não. Se as informações não são registradas adequadamente ou se são omitidas, o profissional de saúde pode não conseguir comprovar suas ações. Por isso é importante que todas as informações reunidas pela equipe de saúde a respeito do paciente estejam no documento, cujo conteúdo, reitera-se, é sigiloso. Como prevê o artigo 85 do CEM, é vedado o manuseio e o conhecimento dos prontuários por pessoas não obrigadas ao sigilo profissional ${ }^{5}$, e o médico não deve, de acordo com o artigo 89 do mesmo diploma, liberar cópias do prontuário sob sua guarda, salvo quando autorizado, por escrito, pelo paciente, para atender ordem judicial ou para a sua própria defesa ${ }^{5}$.

Segundo Thomas ${ }^{6}$, registros médicos devem ser adequadamente guardados com pelo menos dois propósitos: ajudar a avaliar cientificamente o perfil do paciente, por meio da análise de resultados do tratamento e planejamento de protocolos; e ajudar a planejar estratégias governamentais na área da saúde. Trata-se, desse modo, de documento de relevância individual e coletiva. O preâmbulo da Resolução CFM 1.638/2002 considera o prontuário valioso para o paciente, para o médico que o assiste e para as instituições de saúde, bem como para o ensino, a pesquisa e os serviços públicos de saúde, além de [ser] instrumento de defesa legal ${ }^{3}$.

A qualidade do prontuário médico está intimamente relacionada à comunicação. Como apontam Mesquita e Deslandes, esse é documento de construção coletiva, que implica múltiplas ações de registro especializado em um universo de linguagens e de relações complexas entre os saberes e as práticas dos profissionais que integram as equipes de saúde, no sentido de dar transparência aos procedimentos realizados na assistência aos usuários dos serviços, melhorando a qualidade e a eficácia da atenção ao paciente ${ }^{7}$.

Visto que a comunicação eficiente é fundamental, muitas instituições têm tentado modernizar seus serviços adotando, por exemplo, o prontuário eletrônico do paciente (PEP). De acordo com Martins e Lima, o PEP proporciona inúmeras vantagens, entre as quais: agilidade no acesso à informação, intercâmbio de informações, economia de espaço, redução de consumo com impressos, informações gerenciais rápidas e precisas e aumento de tempo para os profissionais se dedicarem aos pacientes ${ }^{8}$, garantindo ainda a legibilidade dos dados registrados. Contudo, os autores apontam algumas desvantagens, como o custo elevado na implantação (equipamentos e treinamentos), a possibilidade do sistema ficar inoperante e a 
resistência da equipe ${ }^{8}$. Com isso, este estudo tem por objetivo avaliar o preenchimento de prontuários médicos em um hospital de ensino, visando verificar se essa instituição possui registros completos e com informações bem apresentadas.

\section{Método}

Foram avaliados 191 prontuários datados a partir de 2016 relativos a pacientes que já tiveram alta. Um hospital de ensino da cidade de Mogi das Cruzes/SP cedeu todos os prontuários. Analisaram-se formulários das áreas de medicina interna do adulto, pediatria e ginecologia-obstetrícia, levando em conta os seguintes itens: identificação do paciente, anamnese, exame físico, diagnóstico, conduta e identificação do profissional. O prontuário consolidado também foi avaliado, por meio do item "fichas destinadas ao preenchimento do médico".

$\mathrm{Na}$ avaliação, aplicou-se questionário estabelecido por Silva e Tavares Neto ${ }^{9}$, resultando em um escore total formado pela soma dos escores parciais relativos ao preenchimento de cada um dos itens do documento. Optou-se por esse instrumento pela abrangência do seu estudo original, que analisou prontuários de 105 instituições filiadas à Associação Brasileira de Hospitais Universitários e Entidades de Ensino ${ }^{9}$. Além disso, o questionário contempla todos os elementos que devem constar do prontuário, em qualquer suporte (eletrônico ou físico), conforme definido no artigo 5o da Resolução CFM 1.638/2002³ :

a. identificação do paciente - nome completo, data de nascimento (dia, mês e ano com quatro dígitos), sexo, nome da mãe, naturalidade (indicando o município e o estado de nascimento), endereço completo (nome da via pública, número, complemento, bairro/distrito, município, estado e CEP); b. anamnese, exame físico, exames complementares solicitados e seus respectivos resultados, hipóteses diagnósticas, diagnóstico definitivo e tratamento efetuado;

c. evolução diária do paciente, com data e hora, discriminação de todos os procedimentos aos quais o mesmo foi submetido e identificação dos profissionais que os realizaram, assinados eletronicamente quando elaborados e/ou armazenados em meio eletrônico;

d. nos prontuários em suporte de papel é obrigatória a legibilidade da letra do profissional que atendeu o paciente, bem como a identificação dos profissionais prestadores do atendimento. São também obrigatórias a assinatura e o respectivo número do CRM;

e. nos casos emergenciais, nos quais seja impossivel a colheita de história clínica do paciente, deverá constar relato médico completo de todos os procedimentos realizados e que tenham possibilitado o diagnóstico e/ou a remoção para outra unidade.

No instrumento de Silva e Tavares Neto ${ }^{9}$, as pontuações para cada item variam de 0 a 4 , sendo o valor nulo atribuído caso a informação não exista ou não haja espaço para seu registro no documento. A pontuação final é resultado da somatória dos escores parciais. No presente estudo consideraram-se "consolidados" os prontuários destinados ao preenchimento de médicos. As fichas preenchidas por outros profissionais da equipe não foram analisadas. Cada variável categórica foi descrita pela frequência e analisada pelo teste qui-quadrado. As variáveis quantitativas sem distribuição normal ou do tipo discreta foram descritas pela média ( \pm desvio-padrão) e mediana, e analisadas pelo teste de Mann-Whitney, comparando os respectivos rankings médios. $O$ resultado foi considerado significativo quando a probabilidade $(p)$ de ocorrência de erro do tipo I (alfa) foi menor que $5 \%$ (ou $p$-valor $<0,05$ ). A Tabela 1 apresenta os resultados encontrados.

Tabela 1. Estatística descritiva para dados obtidos de prontuários médicos considerando o instrumento de Silva e Tavares Neto ${ }^{9}$ em um hospital-escola (Mogi das Cruzes/SP, Brasil, 2016-2019)

\begin{tabular}{|l|l|c|c|c|c|c|c|c}
\hline & & $\mathbf{n}$ & $\boldsymbol{\mu}$ & Mediana & $\boldsymbol{\sigma}$ & EPM & Min. & Máx. \\
\hline \multirow{4}{*}{ Medicina interna } & Ginecologia e obstetrícia & 27 & 46,2 & 48 & 6,48 & 1,250 & 28 & 53 \\
\cline { 2 - 9 } & Medicina interna do adulto & 123 & 41,8 & 43 & 8,35 & 0,753 & 14 & 61 \\
\cline { 2 - 9 } & Pediatria & 41 & 45,5 & 45 & 7,25 & 1,130 & 29 & 57 \\
\hline \multirow{4}{*}{ Prontuário consolidado } & Ginecologia e obstetrícia & 27 & 13,5 & 13 & 1,60 & 0,308 & 10 & 16 \\
\cline { 2 - 9 } & Medicina interna do adulto & 123 & 11,4 & 12 & 2,53 & 0,228 & 3 & 18 \\
\cline { 2 - 9 } & Pediatria & 41 & 11,6 & 11 & 2,50 & 0,390 & 5 & 17 \\
\hline \multirow{4}{*}{ Pontuação geral } & Ginecologia e obstetrícia & 27 & 59,7 & 62 & 6,79 & 1,310 & 42 & 67 \\
\cline { 2 - 9 } & Medicina interna do adulto & 123 & 53,2 & 55 & 9,51 & 0,858 & 24 & 78 \\
\cline { 2 - 9 } & Pediatria & 41 & 57 & 58 & 8,69 & 1,360 & 36 & 71 \\
\hline
\end{tabular}

n: número de observações; $\mu$ : média amostral; $\sigma$ : desvio-padrão; EPM: erro-padrão da média; mín.: mínimo; máx.: máximo 
A partir dos dados coletados, verificou-se a presença de outliers, e não foram detectados dados discrepantes que pudessem gerar vieses. As variáveis dependentes foram: pontuação da medicina interna (PMI), pontuação dos prontuários consolidados (PPC) e pontuação geral (PG). Por se caracterizarem como contínuas-discretas, isto é, assumirem somente valores inteiros, tais variáveis são dados de contagem e não apresentam distribuição de probabilidade gaussiana (normalidade), sendo por isso testadas quanto à normalidade de distribuição. Utilizaram-se os testes de Shapiro-Wilk, Anderson-Darling, Cramér-von Mises e Lilliefors (Kolmogorov-Smirnov), e o gráfico de densidade de probabilidade foi observado. Os testes baseiam-se em confrontar a hipótese de nulidade $\left(\mu_{\text {dados }}=\mu_{\text {normal }}\right)$ com a alternativa $\left(\mu_{\text {dados }} \neq \mu_{\text {normal }}\right)$. Como foi obtido valor de $p<0,05$ (teste de normalidade Shapiro-Wilk com valor de $p=0,0004352$ ), a hipótese alternativa foi aceita, e os dados do presente trabalho diferem de uma distribuição normal fictícia. Logo, os valores de todas as variáveis não são normais.

Foram utilizados dois testes não paramétricos, Kruskal-Wallis e Mann-Whitney, comparando os respectivos rankings médios. Pelo teste de Kruskal-Wallis é possível observar que há diferença entre os setores para todas as variáveis (PMI, PPC e PG). Como o valor de $p$ foi menor que 0,05 $(p=0,0003463)$, a hipótese alternativa de que as medianas entre os setores para as pontuações PMI, PPC e PG diferem entre si foi aceita. Para detalhar ainda mais os resultados, realizou-se o teste de
Mann-Whitney pairwise. Diferenças significativas das medianas entre os setores do hospital (valor de $p<0,05)$ na pontuação geral foram encontradas entre ginecologia-obstetrícia e medicina interna do adulto (valor de $p=0,0001284$ ) e entre pediatria e medicina interna do adulto (valor de $p=0,03569$ ).

Este trabalho está em conformidade com a Resolução CNS $466 / 2012^{10}$ e com os princípios da Declaração de Helsinki ${ }^{11}$.

\section{Resultados}

Os prontuários fornecidos pelo hospital de ensino eram do tipo convencional, não eletrônico. Foram analisados 123 formulários de medicina interna do adulto, 27 de ginecologia-obstetrícia e 41 de pediatria, totalizando 191 prontuários. A pontuação esperada era de 73 pontos para todas as áreas, mas o máximo observado foi de 78 para medicina interna do adulto, 71 para ginecologia-obstetrícia, e 67 para pediatria.

As médias encontradas na pontuação geral dos formulários de ginecologia e obstetrícia, medicina interna do adulto e pediatria foram, respectivamente, 59,7; 53,2; e 57, e as medianas foram, respectivamente, 62,55 e 58 . Portanto, houve pouca diferença entre as médias e medianas encontradas, principalmente nos formulários de pediatria. A Tabela 2 apresenta as médias e medianas dos setores do hospital-escola analisado e seus respectivos valores de $p$.

Tabela 2. Médias e medianas referentes aos resultados encontrados nas pontuações dos setores do hospital-escola (Mogi das Cruzes/SP, Brasil, 2016-2019)

\begin{tabular}{|l|c|c|c|c|c|c|}
\hline \multirow{2}{*}{ Setores do hospital } & \multicolumn{2}{|c|}{ Pontuação da medicina interna } & Pontuação do prontuário consolidado & \multicolumn{2}{|c|}{ Pontuação geral } \\
\cline { 2 - 7 } & Média (DP) & Mediana** & Média (DP) & Mediana & Média (DP) & Mediana \\
\hline $\begin{array}{l}\text { Ginecologia e } \\
\text { obstetrícia ( } n=27)\end{array}$ & $46,2(6,48)$ & $48^{\mathrm{a}}$ & $13,5(1,60)$ & $13^{\mathrm{a}}$ & $59,7(6,79)$ & $62^{\mathrm{a}}$ \\
\hline $\begin{array}{l}\text { Medicina interna } \\
\text { do adulto }(n=123)\end{array}$ & $41,8(8,35)$ & $43^{\mathrm{b}}$ & $11,4(2,53)$ & $12^{\mathrm{b}}$ & $53,2(9,51)$ & $55^{\mathrm{b}}$ \\
\hline Pediatria $(n=41)$ & $45,5(7,25)$ & $45^{\mathrm{a}}$ & $11,6(2,50)$ & $11^{\mathrm{b}}$ & $57(8,69)$ & $58^{\mathrm{a}}$ \\
\hline Valor de $p^{*}$ & \multicolumn{2}{|c|}{0,004} & 0,00007 & 0,0003 \\
\hline
\end{tabular}

*Teste de Kruskal-Wallis; **Teste de Mann-Whitney; letras " $a$ " e " $b$ " na mesma coluna diferem entre si com nível de significância 95\%; DP: desvio-padrão

\section{Discussão}

O hospital de ensino se mostrou solícito em participar do estudo, o que talvez se deva ao crescente interesse quanto ao correto registro dos serviços prestados na área da saúde ${ }^{12}$, bem como ao fato de que a instituição tem como uma de suas finalidades formar novos profissionais ou trabalhar na educação permanente de profissionais já atuantes. Prova de que o tema abordado tem recebido cada vez mais atenção é a obrigatoriedade, imposta pelo $\mathrm{CFM}^{3}$, da Comissão de Revisão de Prontuários 
nas instituições de saúde. Compete a essa comissão observar se todos os itens necessários constam do prontuário e assegurar o preenchimento, a guarda e o manuseio correto dos registros, o que cabe ao médico assistente, à chefia da equipe, à chefia da clínica e à direção técnica da unidade.

As pontuações médias atingidas - de 53,2 para medicina interna do adulto, 59,7 para ginecologia-obstetrícia, e 57 para pediatria ficaram abaixo dos 73 pontos esperados, o que demonstra problemas de qualidade nos prontuários médicos avaliados. Essas lacunas podem prejudicar não somente a qualidade da assistência prestada ao paciente, mas também as atividades de ensino e pesquisa, além de gerar riscos no âmbito legal e impedir que dados relevantes informem políticas públicas.

Com relação a itens específicos, é preocupante observar que informações sobre anamnese e exame físico estavam ausentes ou incompletas. A anamnese é parte essencial da história clínica registrada em prontuário, e é por ela que o médico se guia no exame físico - de suma importância para que a equipe formule ou confirme hipóteses diagnósticas. Quando a anamnese não é bem realizada, todo o cuidado do paciente fica comprometido.

Inspiram preocupação ainda as fichas destinadas ao preenchimento da equipe, indicadas pelos itens "evolução da enfermagem", "fisioterapia", "serviço social", "psicologia" e "terapia ocupacional". A maioria dos prontuários apresentou escore 1 ("presente") com relação à "evolução da enfermagem", mas nos demais itens os resultados não foram satisfatórios. Esses dados indicam pouco atendimento por parte dessas equipes, seja por falta de encaminhamento ou de profissionais da área no hospital, resultando em má comunicação entre a equipe multidisciplinar e afetando o cuidado com o paciente.

Também foram detectadas falhas em dados de epidemiologia, imunização, antecedentes de endemias, acidentes ou violências e antecedentes fisiológicos. Na maioria dos prontuários, esses itens não foram contemplados, recebendo escores baixos ou até mesmo nulos, indicativos de ausência.

Em suma, a análise observou problemas de forma e conteúdo em muitos prontuários. Apesar de a média dos escores obtidos não ser tão baixa, as lacunas em algumas variáveis específicas são preocupantes. A análise dos prontuários possibilita uma leitura crítica sobre as práticas que os profissionais internalizam e reproduzem a partir de sua formação, assim como sobre as condições e circunstâncias operacionais que Ihes são apresentadas e às quais, por inúmeras razões, acabam por se submeter ${ }^{13}$. Assim, seria importante estruturar os prontuários com campos obrigatórios que padronizassem o documento independentemente das diversas formações dos profissionais que compõem a equipe hospitalar.

Cabe apontar que o hospital pesquisado, até o momento da coleta de dados, ainda não havia implantado o PEP, que poderia agilizar o preenchimento do documento, liberando médico e equipe hospitalar para dedicar mais tempo ao cuidado do paciente. Além disso, o prontuário eletrônico facilitaria pesquisas como esta, que visam analisar a qualidade do documento. Destaca-se, porém, que a implantação do PEP exigiria também mudança cultural, pois a gestão deve procurar identificar as vantagens e as desvantagens para a organização, assegurando o processo dentro da instituição ${ }^{8}$. Trata-se, portanto, de processo que demanda considerável investimento humano e financeiro, em razão da necessidade da implantação da tecnologia de informação e de treinamentos da equipe multiprofissional $^{8}$.

\section{Considerações finais}

De acordo com publicação do Conselho Regional de Medicina de São Paulo, o prontuário médico, na verdade prontuário do paciente, é o conjunto de documentos padronizados, ordenados e concisos, destinados ao registro de todas as informações referentes aos cuidados médicos e paramédicos prestados ao paciente ${ }^{14}$. A qualidade do atendimento é diretamente influenciada por esse documento, visto que todas as informações necessárias para a prática assistencial devem estar nele registradas, de modo organizado e claro para facilitar o entendimento e a troca de informações entre a equipe multiprofissional.

Dada a importância do prontuário, apesar de os escores alcançados na análise não terem sido tão baixos, conclui-se que melhorias são necessárias. O registro ineficiente gera dificuldades para a produção científica do hospital - visto que vieses podem influenciar a análise - e prejudica o encaminhamento, já que outros profissionais terão dificuldade de acompanhar a evolução do paciente. Todos esses fatores dificultam o diagnóstico e prognóstico e, não havendo mudanças, podem comprometer o desenvolvimento dos alunos e a qualidade do sistema de saúde. 


\section{Referências}

1. Brasil. Ministério da Saúde. Conceitos e definições em saúde [Internet]. Brasília: Ministério da Saúde; 1977 [acesso 14 jul 2020]. p. 9. Disponível: https://bit.ly/2HoTOkH

2. Brasil. Op. cit. p. 11.

3. Conselho Federal de Medicina. Resolução CFM no 1.638, de 10 de julho de 2002. Define prontuário médico e torna obrigatória a criação da Comissão de Revisão de Prontuários nas instituições de saúde. Diário Oficial da União [Internet]. Brasília, p. 184-5, 9 ago 2002 [acesso 17 abr 2017]. Seção 1. Disponível: https://bit.ly/3mcMN5v

4. Tavakoli N, Jahanbakhsh M. Investigation of retention and destruction process of medical records in the hospitals and codifying appropriate guidelines. J Edu Health Promot [Internet]. 2013 [acesso 17 abr 2017];2:17. p. 1. Tradução livre. DOI: 10.4103/2277-9531.112687

5. Conselho Federal de Medicina. Código de ética médica: resolução CFM no 1.931, de 17 de setembro de 2009 (versão de bolso) [Internet]. Brasília: CFM; 2010 [acesso 17 abr 2017]. Disponível: https://bit.ly/3jmXvEL

6. Thomas J. Medical records and issues in negligence. Indian J Urol [Internet]. 2009 [acesso 17 abr 2017];25(3):384-8. DOI: 10.4103/0970-1591.56208

7. Mesquita AMO, Deslandes SF. A construção dos prontuários como expressão da prática dos profissionais de saúde. Saúde Soc [Internet]. 2010 [acesso 17 abr 2017];3(19):664-73. p. 666. DOI: 10.1590/S0104-12902010000300017

8. Martins C, Lima SM. Vantagens e desvantagens do prontuário eletrônico para instituição de saúde. Rev Adm Saúde [Internet]. 2014 [acesso 17 abr 2017];63(16):61-6. p. 62. DOI: 10.5327/Z15191672201400630004

9. Silva FG, Tavares Neto JT. Avaliação dos prontuários médicos de hospitais de ensino no Brasil. Rev Bras Educ Méd [Internet]. 2007 [acesso 17 abril 2017];31(2):113-26. DOI: 10.1590/S010055022007000200002

10. Conselho Nacional de Saúde. Resolução no 466, de 12 de dezembro de 2012. Aprova diretrizes e normas regulamentadoras de pesquisas envolvendo seres humanos. Diário Oficial da União [Internet]. Brasília, no 12, p. 59, 13 jun 2013 [acesso 22 out 2020]. Disponível: https://bit.ly/2KeJPu8

11. Associação Médica Mundial. Declaração de Helsinki [Internet]. Helsinki: AMM; 1964 [acesso 22 out 2020]. Disponível: https://bit.ly/2ZvA4mt

12. Silva FG, Tavares Neto JT. Op. cit. p. 124.

13. Mesquita AMO, Deslandes SF. p. 667.

14. Rosas CF, coordenador. Ética em ginecologia e obstetrícia. 3a ed. São Paulo: Conselho Regional de Medicina do Estado de São Paulo; 2004. (Cadernos Cremesp). p. 31.

\section{Participação dos autores}

Mariana Cuccato Ribeiro, Bruno Skawinski Dalaneze e Mariah Padovan de Oliveira Peruchi coletaram e analisaram os dados e redigiram o manuscrito. Raquel Barbosa Cintra orientou o trabalho e revisou o artigo.

\section{Correspondência}

Bruno Skawinski Dalaneze - Av. Maestro João Baptista Julião, 743, casa 5 CEP 08790-090. Mogi das Cruzes/SP, Brasil.

Mariana Cuccato Ribeiro - Graduanda - mari.cuccato@gmail.com

(iD) 0000-0002-0768-157X

Bruno Skawinski Dalaneze - Graduando-bruno_dalaneze@hotmail.com

(iD) 0000-0002-4207-9859

Mariah Padovan de Oliveira Peruchi - Graduanda - mariahperuchi@hotmail.com

(iD) 0000-0002-4711-0886

Raquel Barbosa Cintra - Mestre - raquelcintra@gmail.com

(iD) $0000-0001-9838-1312$ 